Louisiana State University

LSU Digital Commons

$12-14-2020$

\title{
Optomechanical entanglement at room temperature: A simulation study with realistic conditions
}

\author{
Kahlil Y. Dixon \\ Louisiana State University \\ Lior Cohen \\ Louisiana State University \\ Narayan Bhusal \\ Louisiana State University \\ Christopher Wipf \\ California Institute of Technology \\ Jonathan P. Dowling \\ Louisiana State University
}

See next page for additional authors

Follow this and additional works at: https://digitalcommons.Isu.edu/physics_astronomy_pubs

\section{Recommended Citation}

Dixon, K., Cohen, L., Bhusal, N., Wipf, C., Dowling, J., \& Corbitt, T. (2020). Optomechanical entanglement at room temperature: A simulation study with realistic conditions. Physical Review A, 102 (6) https://doi.org/ 10.1103/PhysRevA.102.063518

This Article is brought to you for free and open access by the Department of Physics \& Astronomy at LSU Digital Commons. It has been accepted for inclusion in Faculty Publications by an authorized administrator of LSU Digital Commons. For more information, please contact ir@lsu.edu. 


\section{Authors}

Kahlil Y. Dixon, Lior Cohen, Narayan Bhusal, Christopher Wipf, Jonathan P. Dowling, and Thomas Corbitt 


\title{
Optomechanical entanglement at room temperature: A simulation study with realistic conditions
}

\author{
Kahlil Y. Dixon $\odot,{ }^{1}$ Lior Cohen, ${ }^{1}$ Narayan Bhusal $\odot,{ }^{1}$ Christopher Wipf, ${ }^{2}$ Jonathan P. Dowling, ${ }^{1,3,4,5}$ and Thomas Corbitt ${ }^{1}$ \\ ${ }^{1}$ Department of Physics and Astronomy, Louisiana State University, Baton Rouge, Louisiana 70803, USA \\ ${ }^{2}$ LIGO Laboratory, California Institute of Technology, Pasadena, California 91125, USA \\ ${ }^{3}$ NYU-ECNU Institute of Physics at NYU Shanghai, 3663 Zhongshan Road North, Shanghai 200062, China \\ ${ }^{4}$ CAS-Alibaba Quantum Computing Laboratory, CAS Center for Excellence in Quantum Information and Quantum Physics, \\ University of Science and Technology of China, Shanghai 201315, China \\ ${ }^{5}$ National Institute of Information and Communications Technology, 4-2-1, Nukui-Kitamachi, Koganei, Tokyo 184-8795, Japan
}

(Received 9 July 2020; revised 13 October 2020; accepted 25 November 2020; published 14 December 2020)

\begin{abstract}
Quantum entanglement is the key to many applications like quantum key distribution, quantum teleportation, and quantum sensing. However, reliably generating quantum entanglement in macroscopic systems has proven to be a challenge. Here, we present a detailed analysis of ponderomotive entanglement generation in a movable-endmirror-type optomechanical cavity. These cavities utilize optomechanical interactions between the intracavity field and the end mirror to create quantum correlations. We numerically calculate an entanglement measure, the logarithmic negativity, for the quantitative assessment of the entanglement. Experimental limitations, including thermal noise and optical loss, from measurements of an existing experiment were included in the calculation, which is intractable to solve analytically. This analysis shows that lowering optical losses and measurement uncertainties is more important than temperature for observation of the entanglement in movable-end-mirror-type optomechanical cavity experiments. This work will play an important role in the development of ponderomotive entanglement devices.
\end{abstract}

DOI: 10.1103/PhysRevA.102.063518

\section{INTRODUCTION}

Entanglement is the most common and important resource for various quantum technologies, from quantum metrology $[1,2]$ to quantum communication [3] and quantum computing $[4,5]$. It is well known that quantum light sources, in particular entangled sources, require nonlinear interaction. To date, most of these sources are based on all-optical nonlinear processes in crystals [6], which are good enough for most applications but insufficient for applications with very short wavelengths [7]. Recently, efforts have been devoted to exploring different avenues to generate quantum entanglement [8-11]. One approach is to use strong light-matter interaction with single atoms [8] or single quantum dots [9]. While this method is very efficient, its production is limited to a single entangled photon pair at a time. A reliable source of entanglement for short wavelength which provides multiphoton entanglement is still needed.

Radiation pressure - the force electromagnetic radiation exerts on a material surface-is a significant source of noise in optical metrology [12]. The light's momentum causes fluctuations in the mirror's position, yielding phase noise in the electromagnetic wave. However, this interaction creates quantum correlations that can be exploited to produce nonclassical light. It has been shown that when an electromagnetic wave is incident on a mirror, it generates a squeezed light; i.e., the electromagnetic wave experiences an optical nonlinearity $[13,14]$. This nonlinearity can also generate entanglement between the light and the mirror [15-17]. Moreover, for input involving two light sources, an optomechanical cavity can output entangled light [18-22]. This form of bipartite optical entanglement generation has been demonstrated experimentally using a vibrating silicon oxide membrane [23]. This work considers a cantilever micromirror in place of the silicon oxide membrane. This oscillating mirror has higher-order modes that strongly affect entanglement.

To observe the effect of the quantum back-action between the two light fields, we consider a homodyne quadrature variance measurement of two output optical fields from a single-cavity double optical spring with a micromirror. We report the experimental feasibility of observable ponderomotive entanglement in a cantilever-micromirror-type optomechanical cavity at room temperature and lower temperature. This work identifies experimental configurations that will yield observable entanglement using programs, designs, and measurements that have been previously reported [24-26]. We numerically evaluate the amount of entanglement and investigate the dependence of entanglement on various experimental parameters such as temperature, sideband frequency, cavity length, and loss. This work considers such realistic experimental conditions in a computational framework; thus, we predict the efficacy of such an entanglement generation as techniques develop, minimizing losses and noises.

\section{METHODS}

\section{A. Experimental considerations}

The experimental consideration is shown in Fig. 1. We chose this configuration because it allows for a stable 


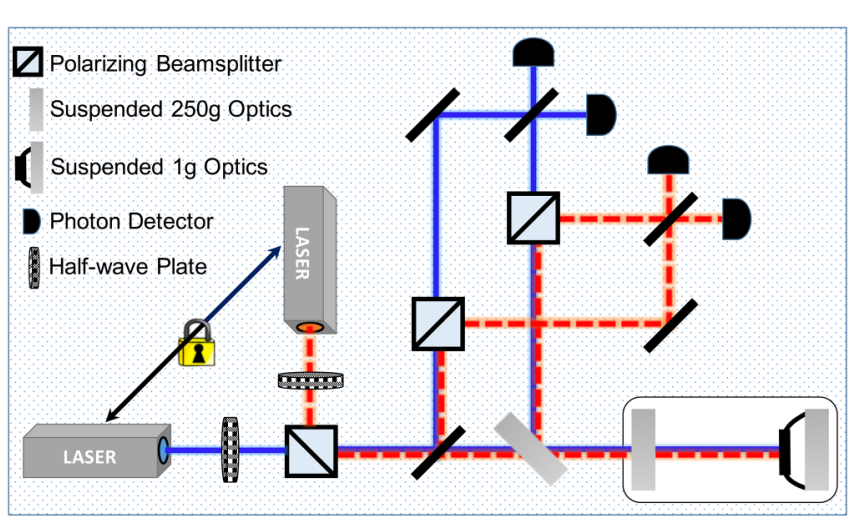

FIG. 1. Schematic diagram of the proposed experiment. The scheme uses two frequency-locked laser fields that are prepared in two orthogonal polarizations before entering the cavity. A balanced homodyne measurement is employed to construct the full quadrature covariance matrix. The scheme utilizes polarizing beam splitters to isolate orthogonal polarizations.

optomechanical system with no external feedback, which could disrupt the entanglement. It utilizes a single optomechanical cavity that acts as a double optical spring. This setup will convert the squeezing effects of the optical spring cavity into entanglement. Measuring this form of entanglement requires dual homodyne detection to properly measure the squeezing correlations. The two lasers are frequency locked to maintain their relative detunings with respect to the cavity resonance. The laser fields are arranged with orthogonal relative polarizations.

To accurately predict entanglement generation from the micromirror cavity, we consider temperature, cavity loss, laser power, and optical spring detunings. Concurrently, we restrict the optical detuning to maintain a stable optical spring, while including more realistic models for input noises. Other variables pertaining to the optomechanical cavity, such as the thermal noise from the micromirror motion (at room temperature), were taken from experimental data of the same setup [25].

\section{B. Measuring entanglement}

To determine whether the output fields are entangled we need to choose a convenient measure. The main measure we use here is the logarithmic negativity entanglement measure $[27,28]$. The variance matrix assembled from the quadrature operators will be the main output to measure. The variance matrix can be written as follows:

$\mathbf{V}=\left(\begin{array}{cccc}\left\langle X_{1}{ }^{*} X_{1}\right\rangle_{+} & \left\langle X_{1}{ }^{*} Y_{1}\right\rangle_{+} & \left\langle X_{1}{ }^{*} X_{2}\right\rangle_{+} & \left\langle X_{1}{ }^{*} Y_{2}\right\rangle_{+} \\ \left\langle Y_{1}{ }^{*} X_{1}\right\rangle_{+} & \left\langle Y_{1}{ }^{*} Y_{1}\right\rangle_{+} & \left\langle Y_{1}{ }^{*} X_{2}\right\rangle_{+} & \left\langle Y_{1}{ }^{*} Y_{2}\right\rangle_{+} \\ \left\langle X_{2}{ }^{*} X_{1}\right\rangle_{+} & \left\langle X_{2}{ }^{*} Y_{1}\right\rangle_{+} & \left\langle X_{2}{ }^{*} X_{2}\right\rangle_{+} & \left\langle X_{2}{ }^{*} Y_{2}\right\rangle_{+} \\ \left\langle Y_{2}{ }^{*} X_{1}\right\rangle_{+} & \left\langle Y_{2}{ }^{*} Y_{1}\right\rangle_{+} & \left\langle Y_{2}{ }^{*} X_{2}\right\rangle_{+} & \left\langle Y_{2}{ }^{*} Y_{2}\right\rangle_{+}\end{array}\right)$,

where $\left\langle u^{*} v\right\rangle_{+}=\frac{\left\langle u^{*} v+v^{*} u\right\rangle}{2}$, or in block form,

$$
\mathbf{V}=\left(\begin{array}{ll}
V_{11} & V_{12} \\
V_{21} & V_{22}
\end{array}\right)
$$

TABLE I. Set of variable simulation parameters, and a stable configuration that yields nonzero entanglement.

\begin{tabular}{lcc}
\hline \hline Parameter & Notation & Stable and $E_{N} \neq 0$ \\
\hline Temperature & $T$ & $295 \mathrm{~K}$ \\
Circulating carrier power & $P_{1}$ & $0.2816 \mathrm{~W}$ \\
Circulating subcarrier power & $P_{2}$ & $0.2238 \mathrm{~W}$ \\
Loss & $L_{s}$ & $250 \mathrm{ppm}$ \\
Carrier detuning & $d_{1}$ & 0.3 \\
Subcarrier detuning & $d_{2}$ & -1.5 \\
Quality factor & $Q$ & 17000 \\
Cavity length & $L_{n}$ & $0.01 \mathrm{~m}$ \\
\hline \hline
\end{tabular}

\section{Logarithmic negativity}

The logarithmic negativity is useful for measuring continuous-variable (CV) entanglement and is monotone for Gaussian beams (see Appendix C for an alternate entanglement measure and results). The information-theoretic meaning of logarithmic negativity in terms of exact entanglement cost of quantum Gaussian states was established in [29,30]. Conveniently, the logarithmic negativity can be calculated from the variance matrix [31]:

$$
E_{N}=\max \left[0,-\ln \sqrt{2 \eta-2 \sqrt{\eta^{2}-4 \operatorname{det} \mathbf{V}}}\right],
$$

where

$$
\eta=\operatorname{det} V_{11}+\operatorname{det} V_{22}-2 \operatorname{det} V_{12} .
$$

\section{Computational resources}

While the quantum Langevin approach is more convenient for an analytical approach, to develop an experimental simulation computationally, sideband operator propagation is preferred due to its more intuitive treatment of the optics and higher modularity [32,33]. The simulation assumes an input field and cavity configuration specified by some parameter configuration $\xi$ and outputs the homodyne measurement of the quadratures. It solves for the output quadratures via successive transformation of the input sideband quadratures. To calculate the effect of the micromirror on the input sidebands, measurement data from previous work with the optomechanical cavity is used to simulate the cantilever's effects. These data allow our simulation to consider the effect of the cantilever's higher-order modes on the entanglement.

These programs are written to calculate the entanglement measures over different parameter spaces. There are nine adjustable parameters, highlighted in Table I. Past experiments identified configurations that would yield observable singlemode squeezing for a single incident beam. These results narrowed our search for optimal parameters (more on these methods in Appendix A).

\section{RESULTS}

The last column in Table I represents a parameter set that generates the highest logarithmic negativity and stable optical spring. After the simulations are performed the output is used to calculate the variance matrices and entanglement measures. All subsequent figures use the parameters in Table I unless 


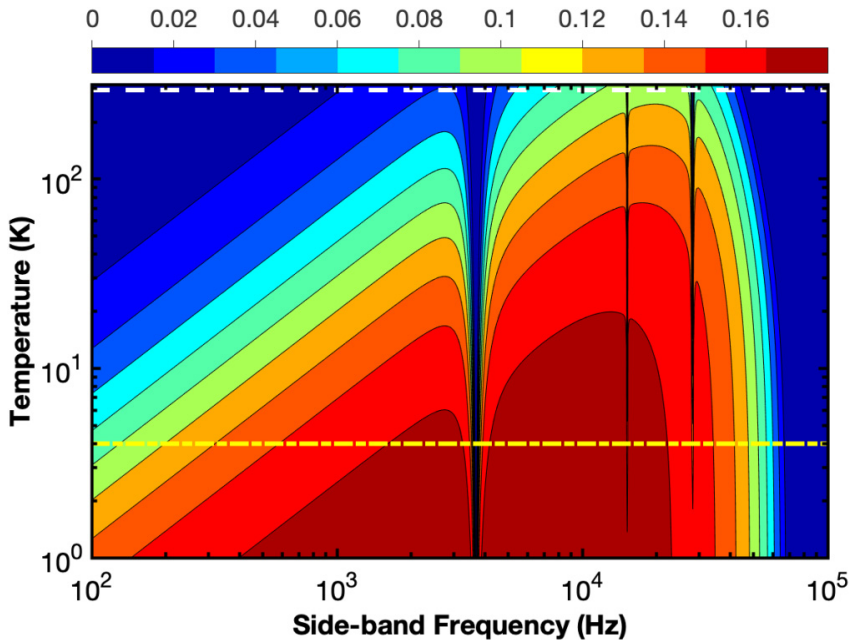

FIG. 2. Logarithmic negativity measure $\left(E_{N}\right)$ of the two output optical fields as a function of temperature and frequency. Conveniently, these parameters yield entanglement at room temperature for a range of frequencies. The sharp drops in $E_{N}$ are due to the higher-order optical spring resonances of the cantilever micromirror (yaw resonance at $4.3 \mathrm{kHz}$ and translation and yaw $54 \mathrm{kHz}$ are the most visible). Top and bottom dotted lines indicate 295 and $4 \mathrm{~K}$, respectively.

otherwise specified. For example, at room temperature and frequency of about $20 \mathrm{kHz}$, the program predicts an output variance matrix $\mathbf{V}$, where $E_{N}(\mathbf{V})=0.104$ and where

$$
\mathbf{V}=\left(\begin{array}{cccc}
17.32 & -51.38 & -21.06 & -14.80 \\
-51.38 & 156.2 & 63.76 & 45.07 \\
-21.06 & 63.76 & 26.61 & 18.47 \\
-14.80 & 45.07 & 18.47 & 13.54
\end{array}\right)
$$

(note that unsqueezed shot noise would have a variance matrix of $\frac{1}{2} \mathbf{I}$, where $\mathbf{I}$ is the identity matrix).

Further analyzing the entanglement yields, we found that the $E_{N}$ was maximum at $20 \mathrm{kHz}$ for the above parameters. This maximum appears to decrease slightly in frequency as temperature decreases, as shown in Fig. 2. In the figure, the sharp drops to zero $E_{N}$ are at the resonance frequencies of higher-order mechanical modes of the cantilever. At these frequencies, the thermal noise spikes and the relative quantum noise is suppressed; consequentially, the $E_{N}$ (and squeezing in Ref. [13]) is strongly suppressed at higher-order frequencies.

Not only is the double optical spring cavity capable of entangling the two fields, it is able to do so at room temperature. Cooling the micromirror increases both the degree of entanglement and the frequencies over which it is produced. However, there is no significant advantage to cooling the micromirror below $4 \mathrm{~K}$, for frequencies of $1 \mathrm{kHz}$ and above. This is a result of the thermal noise being pushed well below the quantum back-action level, as shown in Fig. 4. At about $4 \mathrm{~K}$, the logarithmic negativity maximizes at approximately $E_{N}=0.2$ at frequencies above the yaw resonance at $4.3 \mathrm{kHz}$. Furthermore, the figure shows that the predicted entanglement closely follows the results of the ponderomotive squeezing

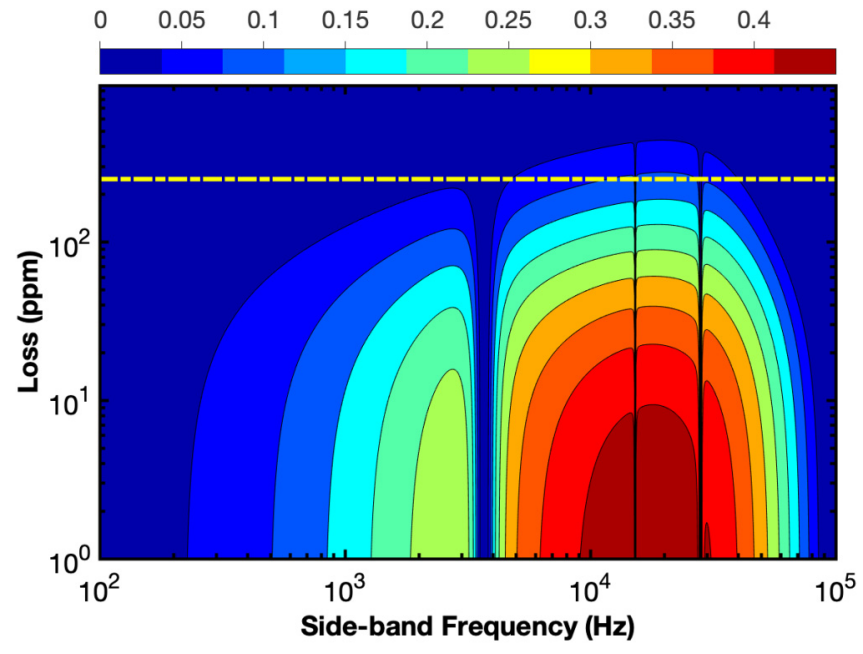

FIG. 3. $E_{N}$ vs loss and frequency at room temperature. For small changes in loss the maximum $E_{N}$ is relatively constant. The three higher-order harmonics are all visible here: yaw, yaw transverse, and roll transverse. The yellow line indicates current experimental losses.

experiment, which also maximized at a frequency of about $20 \mathrm{kHz}$ at room temperature [13].

More promise for this method is inspired by the results displayed in Fig. 3. Even when realistic noise and losses are considered, the entanglement persists. Lower losses also aid the entangler; Fig. 3 shows the entanglement increases as loss decreases. The behavior of the ratio of classical to quantum noise well follows that of the entanglement at all temperatures.

To maximize entanglement, we consider changing the optomechanical cavity length. Figure 4 shows the dependence

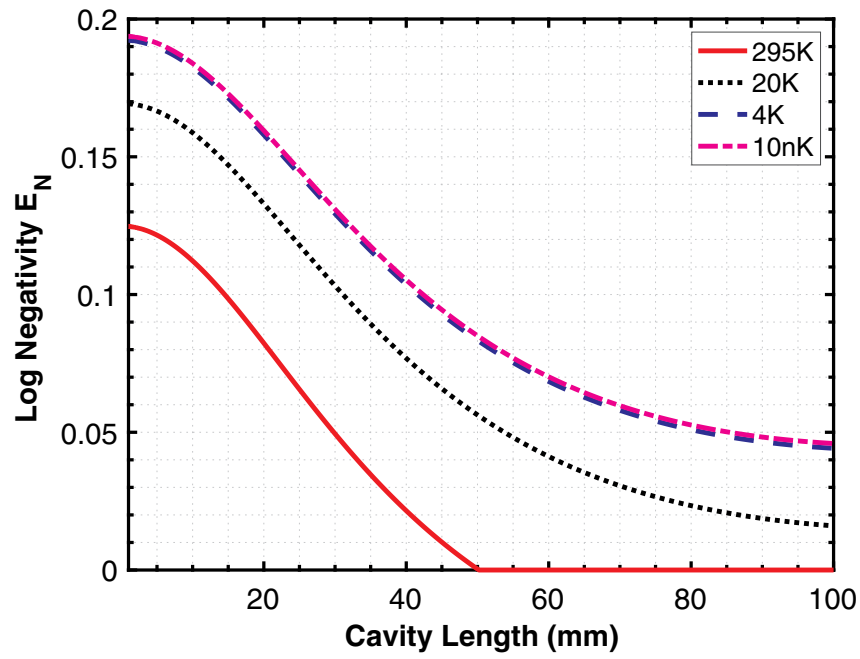

FIG. 4. Logarithmic negativity vs the cavity length for different ambient temperatures at $20 \mathrm{kHz}$. No benefit to entanglement generation will be seen when cooling below $4 \mathrm{~K}$ (unless operating at the cantilever's higher harmonic frequencies). Furthermore, cooling below $1 \mathrm{~K}$ avoids losses to entanglement because harmonic effects of the cavity length effects on the entanglement (the drop at $10 \mathrm{~cm}$ ) are present at all temperatures. Entanglement does not improve at cavity lengths shorter than $1 \mathrm{~mm}$. 


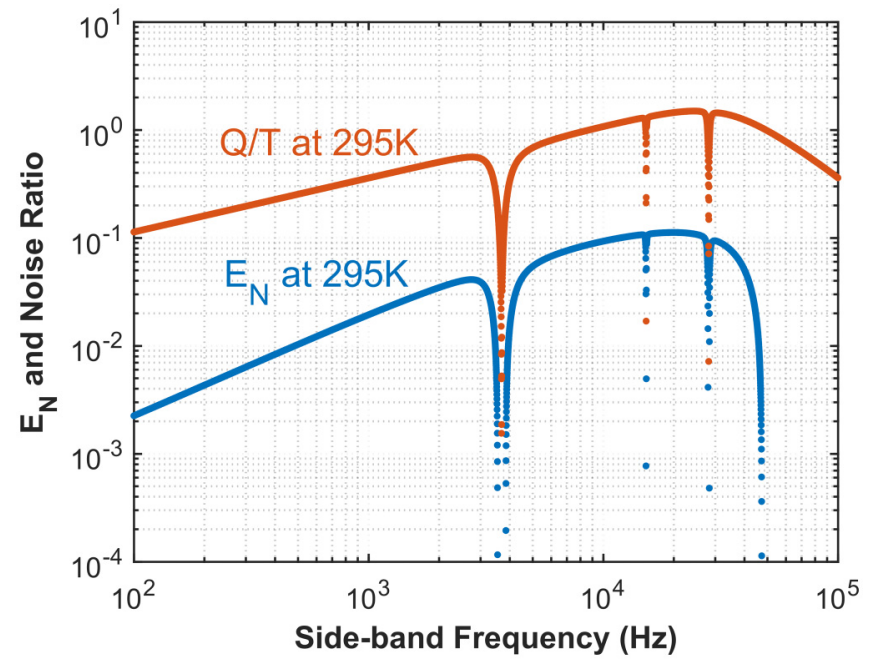

FIG. 5. The ratio of quantum to thermal noises in the system (see Appendix A for calculation). The logarithmic negativity result at room temperature $(295 \mathrm{~K})$ has been included to show agreement. The early decrease of the $E_{N}$ with respect to the noise ratio is due to losses. While this confirms our hypothesis about the quantum radiation pressure noise working in opposition to the thermal noises to yield entanglement, in conjunction with our other results it also shows that such an entanglement generation technique heavily relies on the other experimental parameters as well.

between cavity length and $E_{N}$. Together Figs. 4 and 5 pertain to the fundamental concepts behind optomechanical entanglement generation. The cavity length varies with the input laser power. This input light has quantum fluctuations due to the Heisenberg uncertainty principle. This input quantum noise creates a fundamental uncertainty in the overall cavity length, which in turn strongly affects the properties of the output light. This technique manipulates quantum radiation pressure noise into an entanglement source. When this noise is greater than the classical noise, in this case thermal noise, the entanglement should thrive; Fig. 5 confirms this. Furthermore, entanglement will be limited if the cavity length fluctuations are too small relative to the overall cavity length. Moreover, the dampening effects become more dominant as the cavity length increases, thus widening the resonances that destroy entanglement, as shown in Fig. 4.

We would like to quantify how difficult it is to experimentally verify the existence of the simulated entanglement. We simulate a noisy variance matrix measurement by creating a set of variance matrices normally distributed about the initial output variance matrix at each frequency. (While we only show the experiment's noise sensitivity as a function of Gaussian spread and frequency, it is possible to vary any of the parameters in the table for the noise analysis.) The resulting entanglement uncertainties are plotted in Fig. 6. With measurement certainty on the order of $1 \%$ the output noise in the measurement is several times that of the expected maximum entanglement. When measuring at or near the peak $E_{N}$ frequency, the double homodyne precision must be on the order of $0.1 \%$.

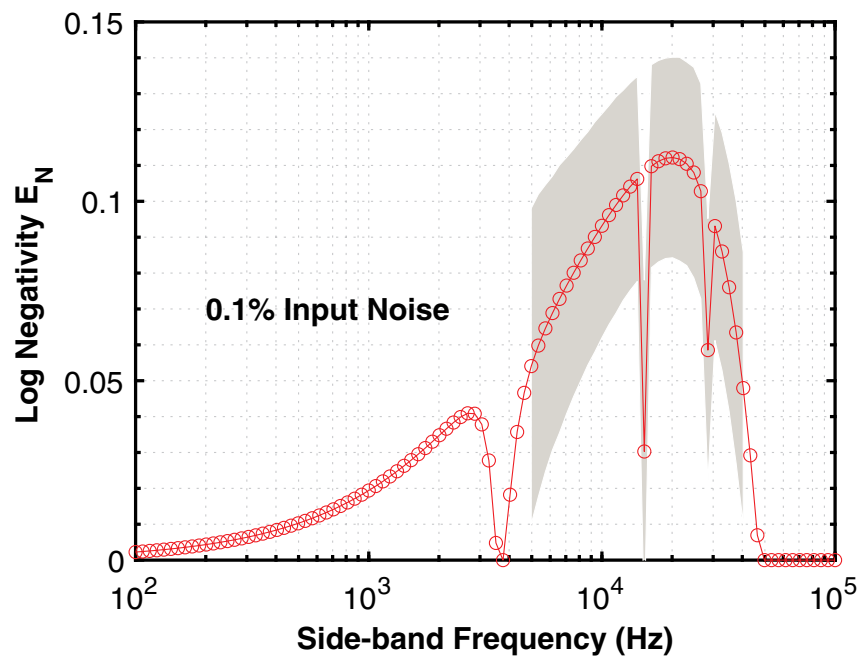

FIG. 6. The effects of Gaussian noise in the double homodyne measurement in the resulting negativity. This was done by creating a normal distribution of variance matrices and calculating the standard deviation. Due to the numeric instabilities in the entanglement measure, entanglement verification requires high-precision measurement of the output quadratures (about $0.1 \%$ ) (67\% confidence interval); the logarithmic negativity is highly sensitive to Gaussian noise. The grey shaded region denotes the uncertainty in the output entanglement. All parameters for this calculation match those in Table I.

\section{CONCLUSION}

All optical circuits and devices are subject to quantum radiation pressure effects. These effects correlate incident light, which implies potential for new entanglement devices. The effects are strong enough to be manipulated into generating bipartite optical entanglement. Moreover, this entanglement persists at room temperature with realistic losses, stable optical spring detunings, and accessible circulating powers. With experimentally stable parameters, we predict a maximum logarithmic negativity versus temperature of $E_{N}=0.2$ in this parameter space; considering parameters close to reported optomechanical entanglement experiments yields an average logarithmic negativity of $E_{N}=0.3$ (with about $1 \%$ measurement certainty) which agrees with the results reported there [23]. Furthermore, we found that entanglement is highly temperature dependent, yet lowering losses is more effective. Lowering losses by two orders of magnitude quadruples the output field's $E_{N}$ which is twice the effect that lowering temperature has on the output entanglement. While lowering losses could enhance entanglement, we have shown that the current loss levels still allow for entanglement. Although predicted entanglement persists despite realistic noise and higher mode considerations, the sensitivity of the system to Gaussian noises presents a significant challenge to experimental realizations. Further optimization may be required to achieve accessible entanglement output in our parameter space.

\section{ACKNOWLEDGMENTS}

K.D., L.C., N.B., and J.P.D. would like to acknowledge the Air Force Office of Scientific Research, Grant No. FA2386-18-1-4010, the Army Research Office, Grant 
No. W911NF-17-1-0541, ARO MURI Grant No. N0001417-S-F006/F47000, the Defense Advanced Research Projects Agency, and the National Science Foundation. We would also like to thank Mark Wilde, Vishal Katariya, and Nicholas Studer for important discussions. This material is based upon work supported by the National Science Foundation under Grant No. PHY-1806634.

\section{APPENDIX A: SIMULATING THE OPTOMECHANICS}

The simulations in this paper used a mathematical framework (which we refer to as the two-photon framework) that developed from the two-photon formalism by Caves and Schumaker. This approach solves for the propagating fields in an interferometer via the input-output equations that pertain to each constituent optic in the interferometer. These methods are described in detail in Ref. [32]. Here we give a more abstract overview of the process.

Let â equal a column vector of sideband annihilation operators in the adapted two-photon formalism specific to our considered experimental setup in Fig. 1 [32]. For our specific experimental setup there is a corresponding matrix $\mathbf{M}$ such that the following is true for each particular parameter configuration $\xi$ :

$$
\mathbf{M a ̂}=\left(\begin{array}{ccc}
M(1,1) & \cdots & M(1,62) \\
\vdots & \vdots & \vdots \\
M(62,1) & \cdots & M(62,62)
\end{array}\right)\left(\begin{array}{c}
\hat{\mathbf{a}}_{1} \\
\vdots \\
\hat{\mathbf{a}}_{62}
\end{array}\right)=\hat{\mathbf{u}},
$$

such that

$$
\hat{\mathbf{u}}=\hat{\mathbf{v}}_{\mathbf{0}}+\hat{\mathbf{l}}_{\mathbf{0}},
$$

where $\hat{\mathbf{v}}_{\mathbf{0}}$ and $\hat{\mathbf{l}}_{\mathbf{0}}$ are column vectors corresponding to vacuum and input laser sidebands. Furthermore, elements of the vector $\hat{\mathbf{a}}$ are the annihilation operators for the sidebands without vacuum noises and laser input. In Fig. 7, the noiseless sidebands are named the critical sidebands. As is explained in the figure, calculating the fields and the homodyne measurement simulation requires the inversion of a $62 \times 62$ matrix. This is one of the differences between the traditional quantum Langevin

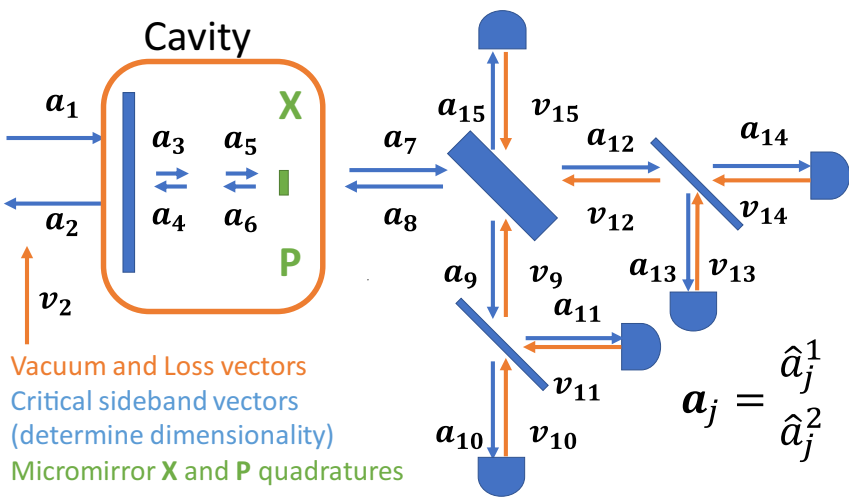

FIG. 7. The program calculates the transformation matrix for the sideband operators $\left(\hat{\mathbf{a}}_{j}\right)$. Since there are 15 fields with each having an upper and lower annihilation operator for a single field input, the program calculates (considering losses and input noises) and inverts a $(30+2) \times(30+2)$ transformation matrix. For the case of two input fields, the matrix is $(60+2) \times(60+2)$. approach and the two-photon reformalism approach. The twophoton methods allow for the development of more robust programs. These programs are more intuitive when including losses, noises, and changes to the experimental apparatus in the computations.

\section{Noise ratio calculation}

This technique solves for all fields in the optical apparatus, so it is convenient for our calculation for the ratio of quantum to thermal noise as well. To begin we assume that the only significant thermal noise source is in the variance of the micromirror's expected position. Next, from the total micromirror displacement we subtract the thermal noise; then we divide by the thermal noise. The results of this calculation for each sideband frequency are graphed in Fig. 5. Since our algorithm solves for all the quadrature fields in Fig. 7, we can extract the total thermal noise from our algorithm by calculating the variance in the $\hat{\mathbf{X}}$ quadrature of the micromirror [34]. Additionally, the optical spring interacts with the intracavity fields, which lowers the effective temperature of the oscillating mirror [35].

\section{APPENDIX B: LOGARITHMIC NEGATIVITY}

Negativity is an "easy-to-compute" measure of entanglement defined as follows:

$$
N(\rho)=\frac{\left\|\rho^{\Gamma_{A}}\right\|-1}{2},
$$

where $\rho$ is the density matrix, $A$ is the dimension of the subsystem, and $\rho^{\Gamma_{A}}$ is the partial transpose of $\rho$ with respect to subsystem $A[36,37]$. Written with the same dependence the logarithmic negativity is the following:

$$
E_{N}=\log _{2}\left\|\rho^{\Gamma_{A}}\right\|_{1} \text {. }
$$

\section{APPENDIX C: DUAN'S MEASURE OF INSEPARABILITY}

Since the logarithmic negativity is strongly dependent on the normalization, we compute a second entanglement measure, Duan's measure, as a sanity check. The comparison of the two measures is shown in Fig. 8. We chose this measure because it does not vary with choice of variance matrix normalization. This entanglement monotone is an alternative to the negativity-based measure for CV entangled beams [38]. The calculation determining the " $a$ " parameter is dependent on calculations done on the variance matrix " $V$ "; however, only variance matrices of certain forms can be used [38]. Fortunately, Duan proved that nonstandard-form variance matrices can be transformed into their standard forms following a few steps and solving a few equations. The variance matrix of the standard form (the goal) is written as follows:

$$
V^{\prime \prime}=\left(\begin{array}{cccc}
n_{1} & & c_{1} & \\
& n_{2} & & c_{2} \\
c_{1} & & m_{1} & \\
& c_{2} & & m_{2}
\end{array}\right) .
$$

These matrix elements are computed from the elements of what we call the "substandard form of the variance matrix"; 


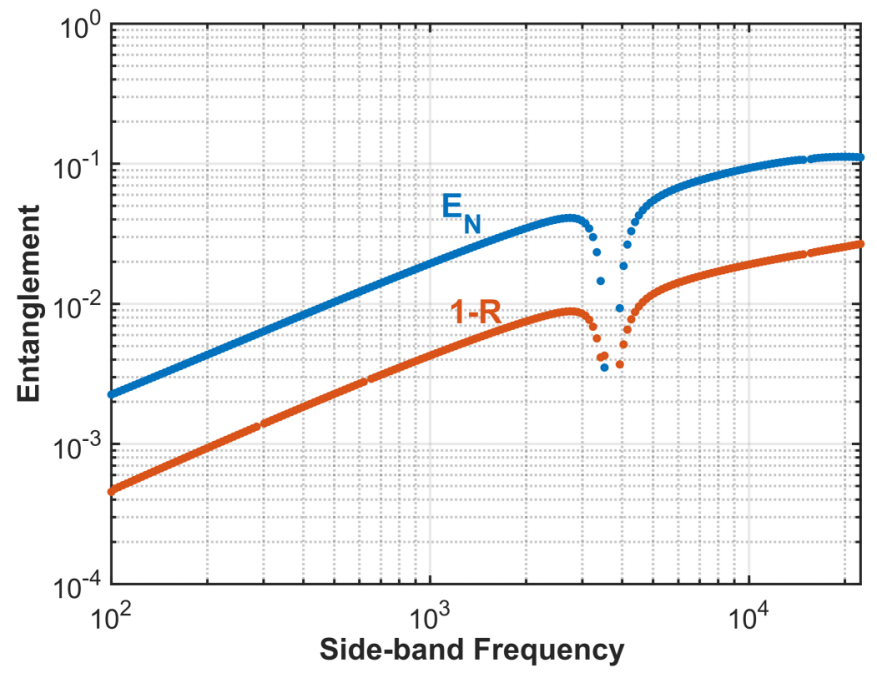

FIG. 8. Duan measure $1-R$ and logarithmic negativity $E_{N}$ vs sideband frequency. For this measure, higher values correspond to stronger entanglement. Both at $T=295 \mathrm{~K}$. The first dip in the Duan measure is due entirely to numeric instabilities from our parameter space. The next is from the instability around the first-order harmonic mode of the oscillator. The further outlying points are due to the instability around the higher-order mode frequencies.

this form is written as follows:

$$
V^{\prime}=\left(\begin{array}{cccc}
n & & c & \\
& n & & c^{\prime} \\
c & & m & \\
& c^{\prime} & & m
\end{array}\right) .
$$

The standard form is calculated from the substandard form by solving the following system of equations for the parameters $r_{1}$ and $r_{2}$ :

$$
\sqrt{r_{1} r_{2}}|c|-\frac{\left|c^{\prime}\right|}{\sqrt{r_{1} r_{2}}}=\sqrt{\alpha_{n} \alpha_{m}}-\sqrt{\left(\beta_{n} \beta_{m}\right)},
$$

$$
\frac{\beta_{n}}{\alpha_{n}}=\frac{\beta_{m}}{\alpha_{m}},
$$

where $\alpha_{n}=n r_{1}-1, \beta_{n}=\frac{n}{r_{1}}-1, \alpha_{m}=m r_{2}-1$, and $\beta_{m}=$ $\frac{m}{r_{2}}-1$. Then, to apply $r_{1}$ and $r_{2}, n_{1}=n r_{1}, n_{2}=n / r_{2}, m_{1}=$ $m r_{1}, m_{2}=m r_{2}, c_{1}=c \sqrt{r_{1} r_{2}}$, and $c_{2}=\frac{c^{\prime}}{\sqrt{r_{1} r_{2}}}$.

Finally, our original variance matrix $V$ is referenced in block form as follows:

$$
V=\left(\begin{array}{ll}
V_{11} & V_{12} \\
V_{12}^{T} & V_{22}
\end{array}\right)=\left(\begin{array}{cc}
A & B \\
B^{T} & C
\end{array}\right) .
$$

Calculating $\mathbf{V}^{\prime}$ from $\mathbf{V}$ was done using the following equations:

$$
\begin{aligned}
& \operatorname{det} A=n^{2}, \quad \operatorname{det} C=m^{2}, \quad \operatorname{det} B=c c^{\prime}, \\
& \operatorname{det} \mathbf{V}=\left(n m-c^{2}\right)\left(n m-c^{\prime 2}\right)
\end{aligned}
$$

After attaining the proper form, the following inequalities need to be broken for there to be entanglement in the system:

$$
\begin{aligned}
& \left|c_{1}\right| \leqslant \sqrt{\left(n_{1}-1\right)\left(m_{1}-1\right)}, \\
& \left|c_{2}\right| \leqslant \sqrt{\left(n_{2}-1\right)\left(m_{2}-1\right)} .
\end{aligned}
$$

To compare Duan's measure with logarithmic negativity, we use the following form of the metric inequality representing Duan's measure:

$$
R=\frac{\frac{a^{2}\left(n_{1}+n_{2}\right)}{2}+\frac{\left(m_{1}+m_{2}\right)}{2 a^{2}}-\left|c_{1}\right|-\left|c_{2}\right|}{a^{2}+a^{-2}} \geqslant 1
$$

where $a^{2}=\sqrt{\frac{m_{1}-1}{n_{1}-1}}$. The system is separable only when this inequality is satisfied. The plot of the two entanglement measures versus the sideband frequency is depicted in Fig. 8.
[1] V. Giovannetti, S. Lloyd, and L. Maccone, Advances in quantum metrology, Nat. Photon. 5, 222 (2011).

[2] A. G. J. MacFarlane, J. P. Dowling, and G. J. Milburn, Quantum technology: The second quantum revolution, Philos. Trans. Roy. Soc. London Ser. A 361, 1655 (2003).

[3] A. K. Ekert, Quantum Cryptography Based on Bell's Theorem, Phys. Rev. Lett. 67, 661 (1991).

[4] R. Raussendorf and H. J. Briegel, A One-Way Quantum Computer, Phys. Rev. Lett. 86, 5188 (2001).

[5] E. Knill, R. Laflamme, and G. J. Milburn, A scheme for efficient quantum computation with linear optics, Nature 409, 46 (2001).

[6] P. G. Kwiat, K. Mattle, H. Weinfurter, A. Zeilinger, A. V. Sergienko, and Y. Shih, New High-Intensity Source of Polarization-Entangled Photon Pairs, Phys. Rev. Lett. 75, 4337 (1995).

[7] S. Sofer, E. Strizhevsky, A. Schori, K. Tamasaku, and S. Shwartz, Quantum Enhanced X-ray Detection, Phys. Rev. X 9 , 031033 (2019).
[8] A. Reiserer, N. Kalb, G. Rempe, and S. Ritter, A quantum gate between a flying optical photon and a single trapped atom, Nature 508, 237 (2014).

[9] H. Wang, H. Hu, T.-H. Chung, J. Qin, X. Yang, J.-P. Li, R.-Z. Liu, H.-S. Zhong, Y.-M. He, X. Ding, Y.-H. Deng, Q. Dai, Y.-H. Huo, S. Höfling, C.-Y. Lu, and J.-W. Pan, On-Demand Semiconductor Source of Entangled Photons Which Simultaneously Has High Fidelity, Efficiency, and Indistinguishability, Phys. Rev. Lett. 122, 113602 (2019).

[10] A. Orieux, M. A. M. Versteegh, K. D. Jöns, and S. Ducci, Semiconductor devices for entangled photon pair generation: A review, Rep. Prog. Phys. 80, 076001 (2017).

[11] O. Alibart, J. Fulconis, G. Wong, S. Murdoch, W. Wadsworth, and J. Rarity, Photon pair generation using four-wave mixing in a microstructured fibre: Theory versus experiment, New J. Phys. 8, 67 (2006).

[12] C. M. Caves, Quantum-Mechanical Radiation-Pressure Fluctuations in an Interferometer, Phys. Rev. Lett. 45, 75 (1980). 
[13] N. Aggarwal, T. J. Cullen, J. Cripe, G. D. Cole, R. Lanza, A. Libson, D. Follman, P. Heu, T. Corbitt, and N. Mavalvala, Room-temperature optomechanical squeezing, Nat. Phys. 16, 784 (2020)

[14] S. Zippilli, G. D. Giuseppe, and D. Vitali, Entanglement and squeezing of continuous-wave stationary light, New J. Phys. 17, 043025 (2015)

[15] C. Genes, A. Mari, P. Tombesi, and D. Vitali, Robust entanglement of a micromechanical resonator with output optical fields, Phys. Rev. A 78, 032316 (2008).

[16] D. Vitali, S. Gigan, A. Ferreira, H. R. Böhm, P. Tombesi, A. Guerreiro, V. Vedral, A. Zeilinger, and M. Aspelmeyer, Optomechanical Entanglement between a Movable Mirror and a Cavity Field, Phys. Rev. Lett. 98, 030405 (2007).

[17] Q. Lin, B. He, and M. Xiao, Entangling Two Macroscopic Mechanical Resonators at High Temperature, Phys. Rev. Appl. 13, 034030 (2020).

[18] C. Wipf, T. Corbitt, Y. Chen, and N. Mavalvala, Route to ponderomotive entanglement of light via optically trapped mirrors, New J. Phys. 10, 095017 (2008).

[19] C. Wipf, Toward quantum opto-mechanics in a gram-scale suspended mirror interferometer, Ph.D. thesis, Massachusetts Institute of Technology, 2013.

[20] Y.-D. Wang and A. A. Clerk, Reservoir-Engineered Entanglement in Optomechanical Systems, Phys. Rev. Lett. 110, 253601 (2013).

[21] S. Barzanjeh, E. S. Redchenko, M. Peruzzo, M. Wulf, D. P. Lewis, G. Arnold, and J. M. Fink, Stationary entangled radiation from micromechanical motion, Nature 570, 480 (2019).

[22] A. Bienfait, K. J. Satzinger, Y. P. Zhong, H.-S. Chang, M.H. Chou, C. R. Conner, É. Dumur, J. Grebel, G. A. Peairs, R. G. Povey, and A. N. Cleland, Phonon-mediated quantum state transfer and remote qubit entanglement, Science 364, 368 (2019).

[23] J. Chen, M. Rossi, D. Mason, and A. Schliesser, Entanglement of propagating optical modes via a mechanical interface, Nat. Commun. 11, 943 (2020).

[24] T. Corbitt, Y. Chen, E. Innerhofer, H. Müller-Ebhardt, D. Ottaway, H. Rehbein, D. Sigg, S. Whitcomb, C. Wipf, and N. Mavalvala, An All-Optical Trap for a Gram-Scale Mirror, Phys. Rev. Lett. 98, 150802 (2007).
[25] S. Sharifi, Y. M. Banadaki, T. Cullen, G. Veronis, J. P. Dowling, and T. Corbitt, Design of microresonators to minimize thermal noise below the standard quantum limit, Rev. Sci. Instrum. 91, 054504 (2020).

[26] J. D. Cripe, Broadband measurement and reduction of quantum radiation pressure noise in the audio band, Ph.D. thesis, Louisiana State University, 2018.

[27] G. Vidal and R. F. Werner, Computable measure of entanglement, Phys. Rev. A 65, 032314 (2002).

[28] M. B. Plenio, Logarithmic Negativity: A Full Entanglement Monotone That Is Not Convex, Phys. Rev. Lett. 95, 090503 (2005).

[29] K. Audenaert, M. B. Plenio, and J. Eisert, Entanglement Cost Under Positive-Partial-Transpose-Preserving Operations, Phys. Rev. Lett. 90, 027901 (2003).

[30] X. Wang and M. M. Wilde, Cost of Quantum Entanglement Simplified, Phys. Rev. Lett. 125, 040502 (2020).

[31] A. Serafini, Quantum Continuous Variables: A Primer of Theoretical Methods, 1st ed. (CRC Press, Boca Raton, FL, 2017), Vol. 1.

[32] T. Corbitt, Y. Chen, and N. Mavalvala, Mathematical framework for simulation of quantum fields in complex interferometers using the two-photon formalism, Phys. Rev. A 72, 013818 (2005).

[33] C. M. Caves and B. L. Schumaker, New formalism for twophoton quantum optics. I. Quadrature phases and squeezed states, Phys. Rev. A 31, 3068 (1985).

[34] V. Giovannetti and D. Vitali, Phase-noise measurement in a cavity with a movable mirror undergoing quantum Brownian motion, Phys. Rev. A 63, 023812 (2001).

[35] T. Corbitt, C. Wipf, T. Bodiya, D. Ottaway, D. Sigg, N. Smith, S. Whitcomb, and N. Mavalvala, Optical Dilution and Feedback Cooling of a Gram-Scale Oscillator to $6.9 \mathrm{mK}$, Phys. Rev. Lett. 99, 160801 (2007).

[36] E. M. Rains, Bound on distillable entanglement, Phys. Rev. A 60, 179 (1999).

[37] S. Bäuml, S. Das, X. Wang, and M. M. Wilde, Resource theory of entanglement for bipartite quantum channels, arXiv:1907.04181.

[38] L.-M. Duan, G. Giedke, J. I. Cirac, and P. Zoller, Inseparability Criterion for Continuous Variable Systems, Phys. Rev. Lett. 84, 2722 (2000). 